observándose que los hábitos de vida, en los tungurahuenses, son poco saluda-
bles; a pesar de que existen politicas pubilicas nacionales tendientes a mejorarlas.

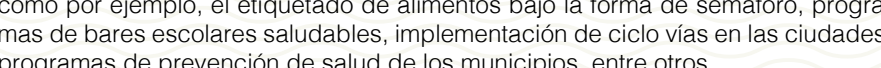

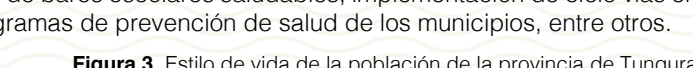

\section{$52,9 \%$ del la población entre 20 y 59
anios consume tabaco}

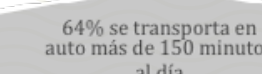

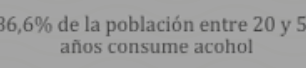

Fuente: Freire, Ramirez-Luzuriaga Belmont Mendieta. Silva-Jaremilo 2004$)$ a doble carga de la mallutriticion: dessutrición y sobrepeso-obesidad, convergen América Latina y el Programa Mundial de Alimentios (CEPAL y PMA, 2017) cuyos 4.346 millones de dólares que en relación al PlB representan el $4,3 \%$; adicional mente genero efectos en la educación, el $32 \%$ de la repitencia escolar fue caus

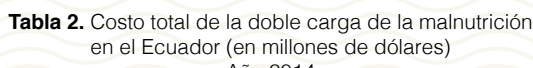

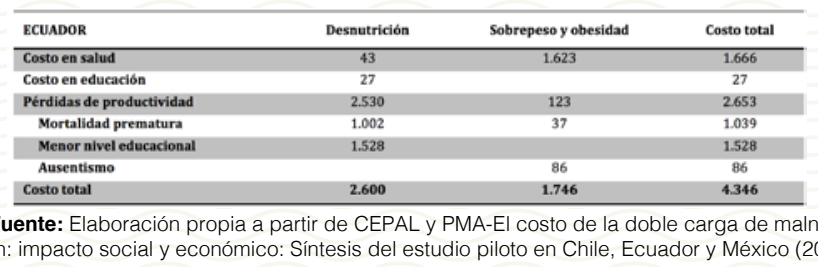
ta Organización de las Naciones Unidas para la Agricultura y la Alimentación

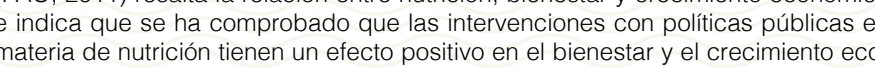
nomico y que aumenta los rendimientos de las inversionese en educación y atención
médica. En el caso del Ecuador el programa de mayor importancia es el deno nado "Acción Nutrición" que está a cargo del Ministerio Coordinador de Desarrololo Social, cuenta con financiamiento del Estado y su proposito es articular estuerzos
para acabar con la malnutricion en el Ecuador

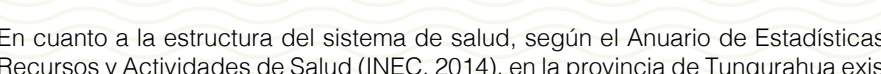
en 155 establecimienilos de salua, de los cuales 30 tienen servicio de internación hospitalara; con enstos datos. Tunguranua se ubica entre las provincias con
considerable nummero de establecimientos de salud. En lo relacionado al persona

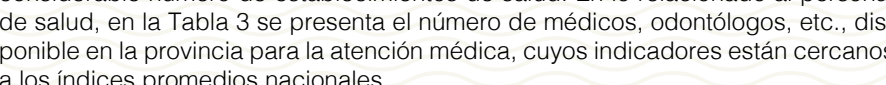

Tabla 3. Tasa de personal de salud en la provinicia de Tungurahu

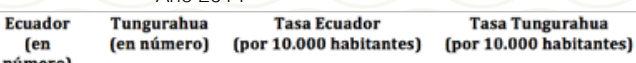

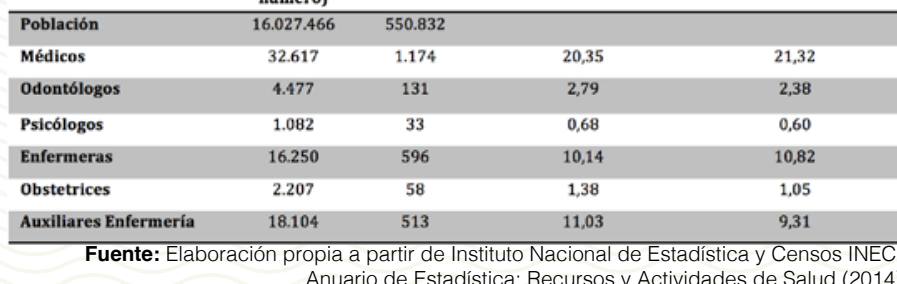

Reflexiones

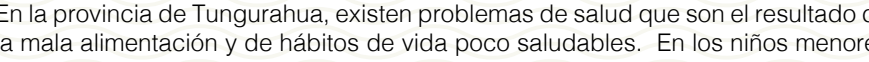
de cinco años la prevalencia de bajo peso y y talla es alarmante; mientras que generativas. Los servicios de salud con los que cuenta la provininia están en los
La configuración de la situación actual de salud en Tungurahua, puede traer como
consecuuencias enormes perdidas por los costos de oportunidad, por los costos

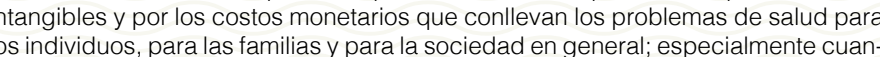
do quienes fallecen son los sostenes económicos del hogar o cuando los gastos en

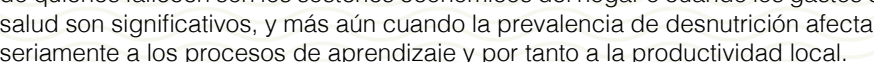

Por tanto, es fundamental que con la finalidad de cuidar y potenciar el capital
humano de la provincial y garantizar su desarrollo económicos se creen a agendas ocales para garantizar la puesta en práctica de politicas nacionales en vigencia. mportante recordar que el crecimiento económico es trascendental pero que el desarrollos social es imperativo para garantizar el bienestara a largo plazo.

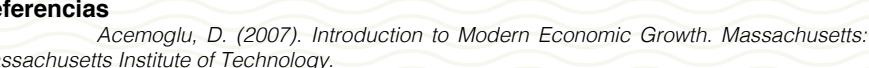
secker, G. (1983). El capital humano. Madrid. Alianzza

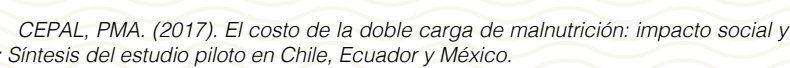

FAO. (2011). El Estadad Munndial de la Agricultura y la Alimentación. Roma: Grupo Cault, M. (2007). Nacimiento de la biboolitica. Buenos Aires.FCE

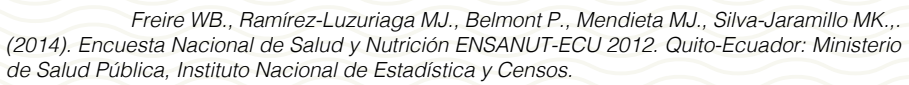

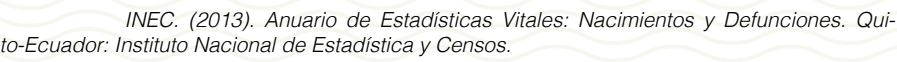
INEC, (2014). Anuario de Estadistica: Recurros y Actividades de Salud. Quito:
Instituto

Mwabu, G. (2007). Health E Eromics for low-ind Tadaro. M. \& Smith S. (2012). Economic Development. Estados Unidos: Pearson

\title{
Los eventos adversos en la provincia de Tungurahua
}

Jorge Tamayo-Viera' Ángel Poaquiza-Poaquizaa ${ }^{2}$ Borman Vargas-Villacres ${ }^{3}$

Fecha de recepción: 16 de Mayo 2017

Resumen

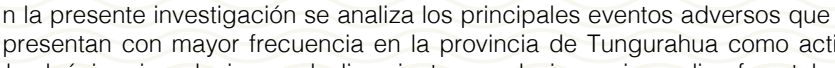

estructurales. Las emergencias se originan por desastres naturales, producidi

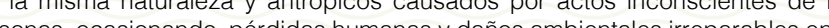
medio ambiente, en un sitio particular y tiempo determinado. Se realiza un análiss

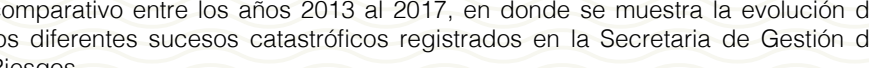

\section{Riesgos.}

we analyze the main adverse events that ocour thesenty in the province of Tungurahua such as volcanic activity, floods, lands sasters, produced by the same nature and anthropic caused by unconscious acts
of people, causing human losses and irvearable environmental damage to the

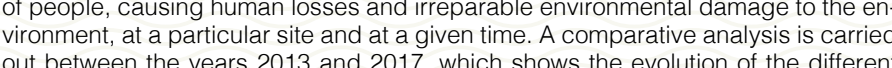

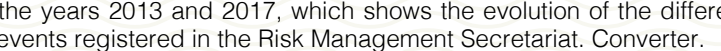
ads: Adverse events. Tungurahua, floods, fires, nature

En la actualdaad nuestro país está expuesto a varios factores de riesgo, ya que nas pobladas. "El Tungurahua es uno de los volcanes más activos del Ec auado continental" "Le Pennec Jean-Luc, 2005, p. 13). De igual manera se producen sis placas Nazza y Sudamericana produce fuerzas muy importantess sobre los bordes tambas placas, se producen fracturas en el interior de ellas, denominandoss os embates causados por la naturaleza, los mismos que han originado daño protección de l a naturaleza, la participación y seguridad de la población, yel orde-

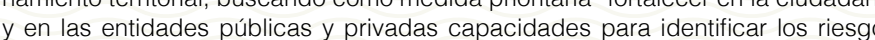
inherentes a sus respectivos ámbitios de acción, informar sobre ellos, ei nnorroora acciones
p. 175).

Previa a la ocurrencia de un determinado evento adverso se activan operativos cia como el servicioi integradodo de seguridad ECU 911 , Ia Secretararia de Gestion d rección de Tránsito, Transporte y Movilidad del Municipio de Ambato. "La gestion de riesgos es responsabilidad directa de cada gobierno autónomo descentraliza.
do odentro de su respectivo âmbito. Cuando sus capacidades para la gestión de riesgoss sean insuricientes, las instancias de mayor jurisdicción territorial y majos

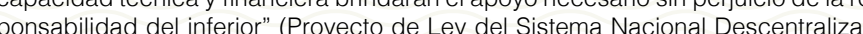
de Gestión de Riesgos, 2013, p. 9), a traves de los comités de gestión de riesgos

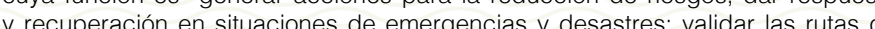
evacuación y zonas seguras, y crear planes de contingencia ante posibles daños"

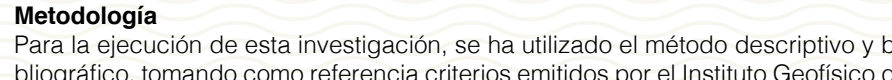
Ia Escuela Politécnica Nacional, relacionando información de las diferentes mag
nitudes sismicas presentadas en los sitios cercanos a los cantones de la proving de Tungurahua. De igual forma se ha tomado información estadistitica proporciona al numero devition al número de eventos adversos registrados en el periodo comprendido entre los Destrucción en el ecosistema y que han alterado las condiciones de vida de la

Fecha de aceptación: 28 de Junio 2017 Resultados

Tabla 1. Porcentiaje de actividad volcánica en la provincia de Tungurahera

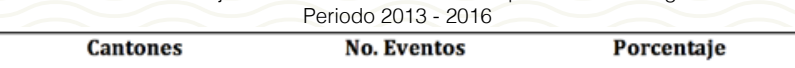
\begin{tabular}{ccc} 
Ambato & 6 & $7,23 \%$ \\
\hline
\end{tabular} \begin{tabular}{ccc} 
Cevallos & 3 & $3,61 \%$ \\
Mocha & 8 & $9,64 \%$ \\
\hline & 2 & $2,4 \%$
\end{tabular} Patate $\quad 22 \quad 2,41 \%$ Pelileo $\quad 22 \quad 26,51 \%$ $\begin{array}{lrr}\text { Quero } & 29 & 34,94 \% \\ \text { Tisaleo } & 8 & 9,64 \%\end{array}$ \begin{tabular}{ccc} 
& 83 & $100,00 \%$ \\
\hline
\end{tabular}

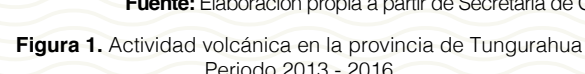

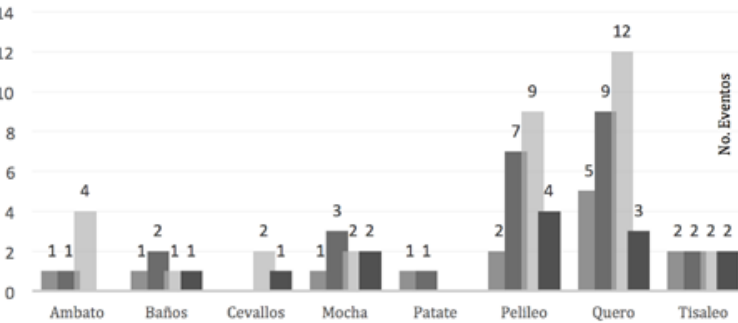
$\begin{array}{lllll}2013 & =2014 & =2015 & * 2015\end{array}$

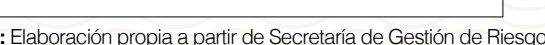
El cantón Baños presenta el mayor número de desizizanientos de tierra con dos-

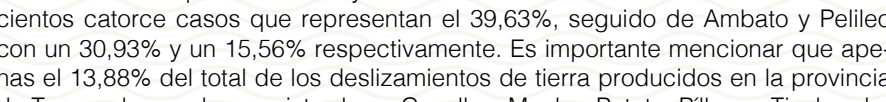
de Tunguratua se han regis
lante el periodo $2013-2017$.
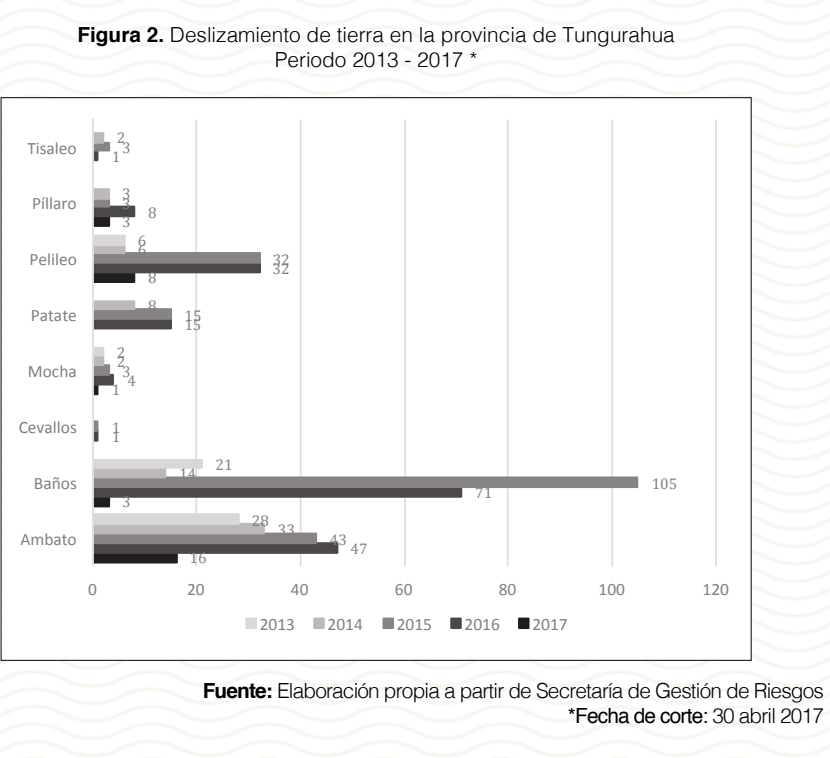

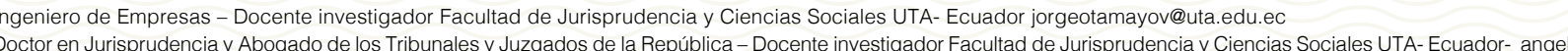

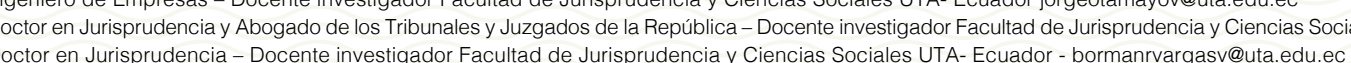


En el cantón Ambato se registra la mayor cantidad de incendios forestales con cin-
cuenta y cinco casos en el año 2013 s seguido de Patate con diez y nueve eventos

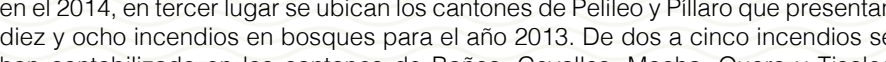
han contabilizado en los cantones de Baños, Cevallos, Mocha, Quero y Tisaleo
para el año 2013 con daños irreparables en la naturalezza producto de la quema

Figura 3. Incendios forestales en la provincia de Tungurahu
Periodo 2013 - 2017

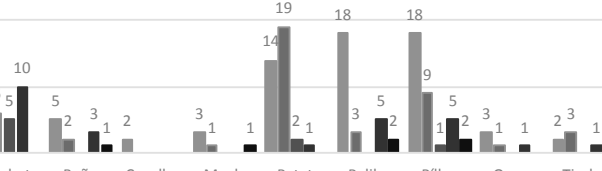

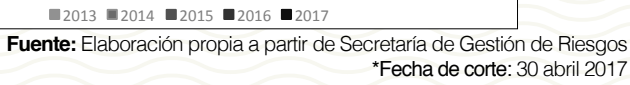

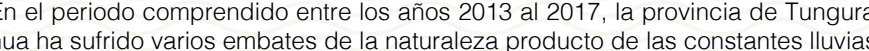
siendo la ciudad de Ambato la aue presenta la mayor rincidencia de casos cond $50 \%$, registrándose diez alertas por inundaciones en el año 2015 , Pelileo presenta
cuatro eventos en el 2016 y en Patate se contabilizan cinco casos de innda ciones en 2015. Pocos casos de inundación se han presentado en los cantones
Cevallos, Quero, isisaleo y Baños. Hasta el 30 de abril del 2017 se registran dos

Figura 4. Inundaciones en la provininia de Tungurahua
Periodo 2013 - $2017^{\star}$

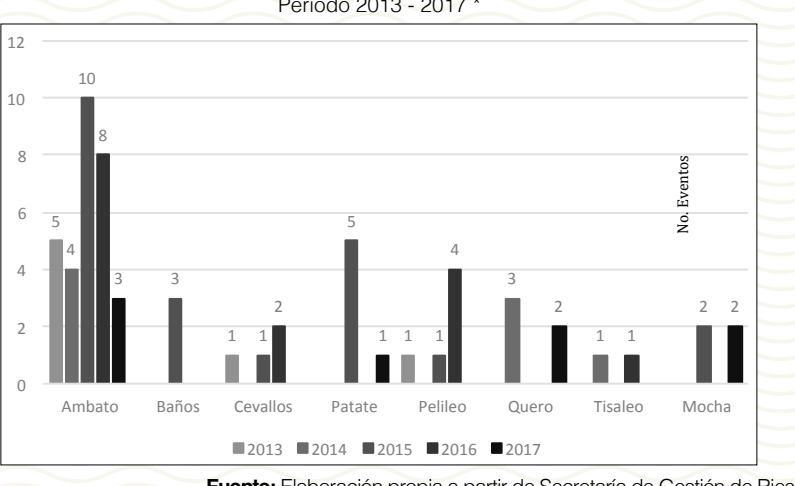

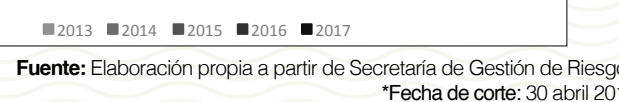

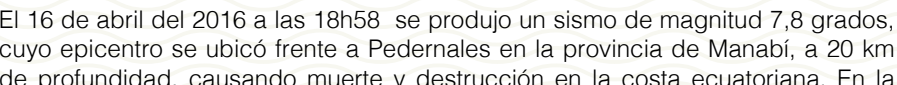
de profundidad, causando muerte y destrucción en la costa ecuatoriana. En
provincia de Tungurahua también se registraron sismos principalmente en Pelilea

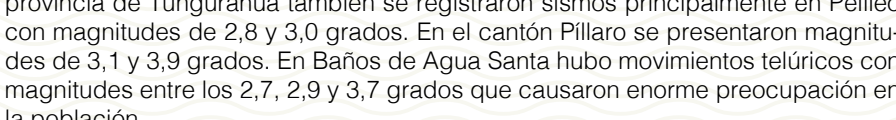
magnitudes

Figura 5. Sismos en la provinciale Tungurahua
Año 2016

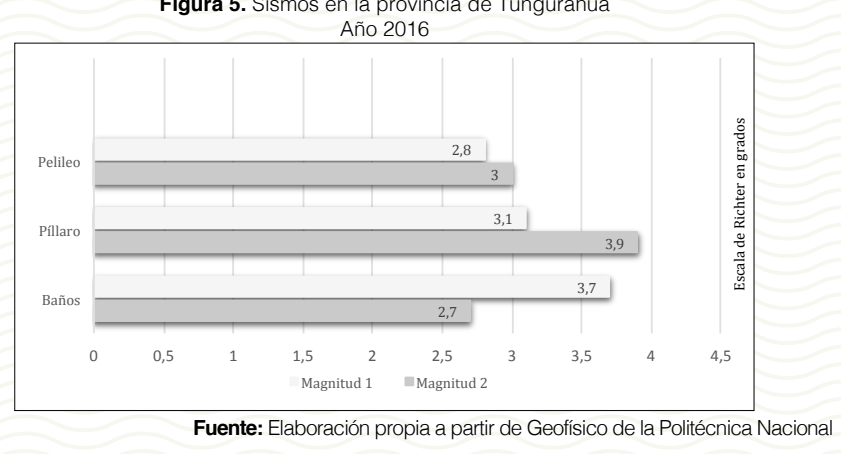

En el año 2016 se registraron dos sismos en la provincia de Tungurahua, siendo los
de mayor magnitud en los cantones de Pelilio,

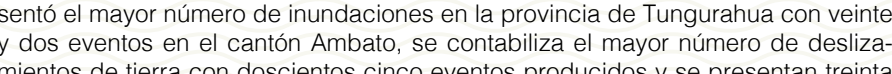

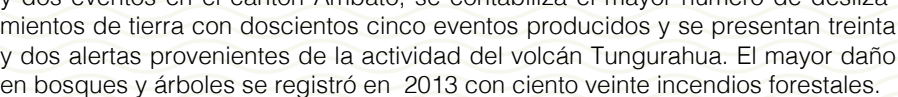

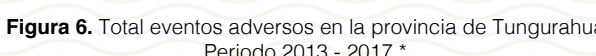

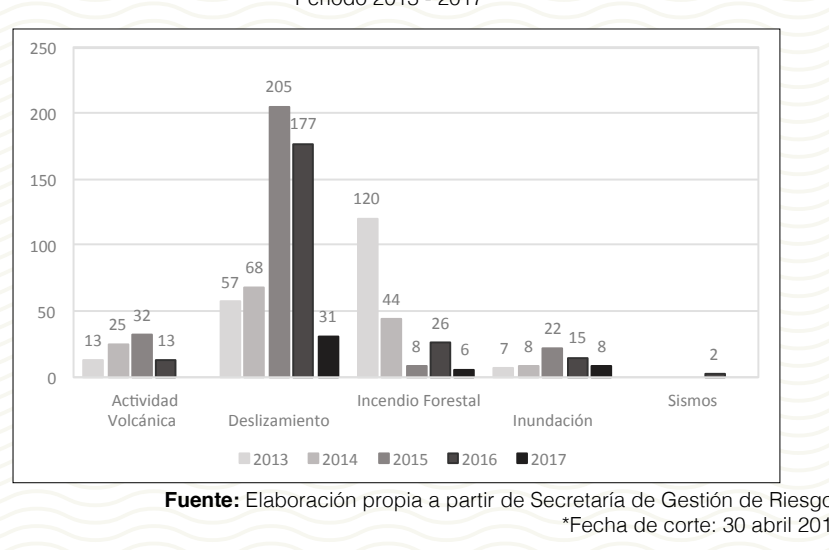

eflexiones

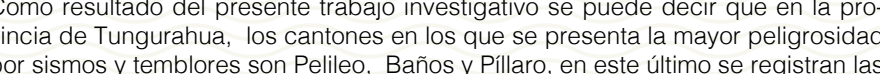
mayores magnitudes sismicas que van desdel los 3,1 a 3,9 grados en l a escala de a zona se ha producido en Baños de Agua Santa con ciento cinco eventos en el año 2015 , conjintamente con Ambato y P Pelier o resultan los cantones más afeccantones de Pelilieo y Ambato han presentado las mayores inundaciones fluviales a conseceuencia de las constantes Iluvias producidas en el año 2016 , es importatrate del total de las inundaciones. Los incendios forestaless de origen natural y antrópico más afectado con consetentala y seis casos prosesentadoos y y equivale al al $37.44 \%$ d del total de incendios registrados en la provincia. Patate y Pillaro constituyen el segundo y rica se ha generado en los cantones de Quero. Pelilieo, Mocha y Ambato ventre el $2013 y 2016$, debiendo mencionarar que los cantones más afectados son Quero con
$2134.94 \%$ y Pellileo con un $26.51 \%$ respectivamente. En los cantones de Ambato

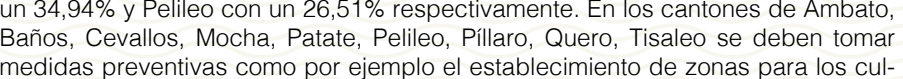

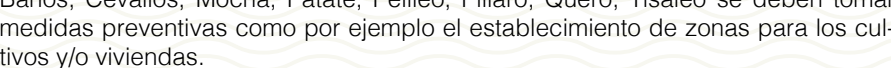

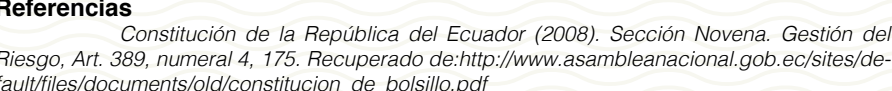

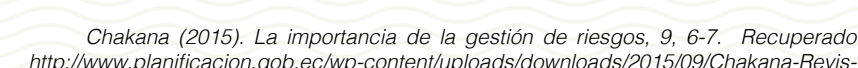

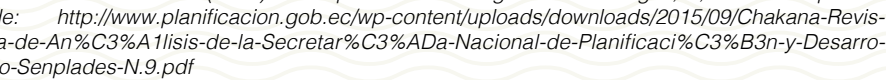

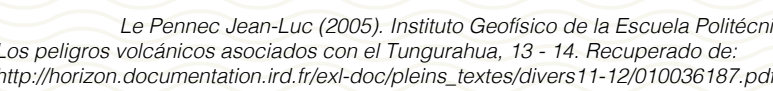

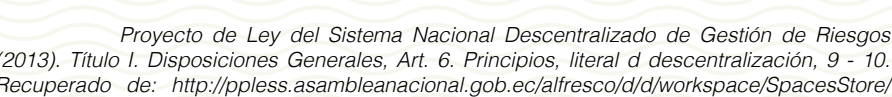

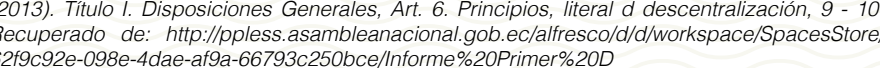

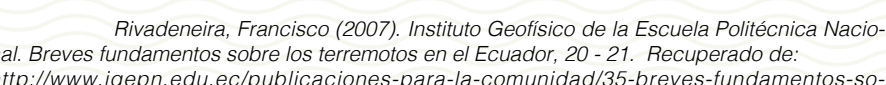

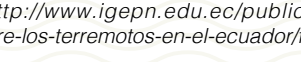

Secretaria de Gestión de Riessoss (2017). Coordinación Zonal/3 de Gestión de Riesgos

\section{Principios tributarios de eficiencia y simplicidad administrativa:}

Caso retenciones en la fuente del Impuesto a la Renta

\section{Eduardo Paredes-Paredes' Gabriela Guevara-Perrazo ${ }^{2}$}

Fecha de receppcion: 14 de Junio 2017

$E^{\text {Resumen }}$

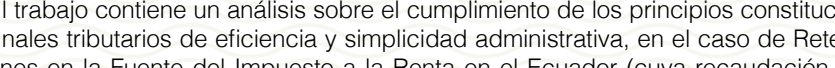
administrada por el Servicio de Rentas Internas - SRI), que se realizan a las personas

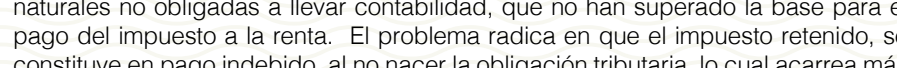
constituye en pago indebido, al no nacer la o obligación tributaria, lo cual acarrea mas
tramitis Palabras clave: Principios, eficienc

The paper contains an analysis of the compliance of the tax constitutional prin-

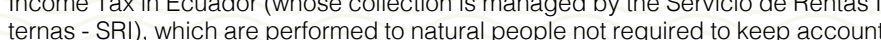
Who have not exceeded the base for the payment of income tax. The problem lies
in the fact that the withholding tax is constituted as an undue payment, since the tax liability does not arise, which entalls more formalities to SRI and other implications
Keywords: Principles, efficiency, simplicity, witholding tax, tax administration

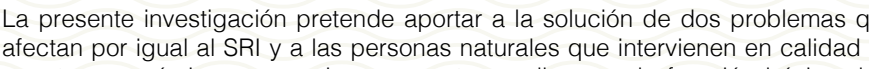
garavada de Imouesto a la Rentat (FBDPR) reclamos de pagos indebidos del Impuesto a a Renta que les ha sido retenido en
la tuente; $y$, , 2) Posibilidad cierta que prescriba la acción de cobro de los citados En cuanto al primer problema mencionado, éste nace en los contribuyentes des ponible de impuesto a la renta, no supera la fracción básica descravada para ef

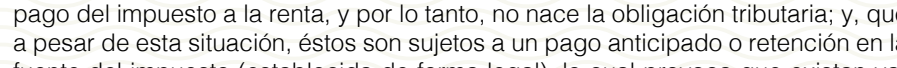
fuente del impuesto (establecida de forma legal), lo cual provoca que existan va lores pagados indebidamente, yse presentenvarios tramities ante el SRi por parte la Administración Tributaria, le exige la i inversión de tiempo y recurs sos econónicos. Asimismo, en lo que respecta al segundo problema en mención, en muchos casos, aquellos contribuyentes que tienen un saldo a tavor por concepto der retenciones

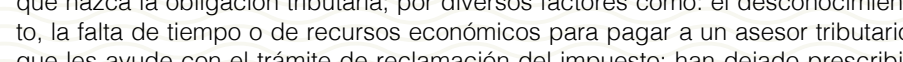

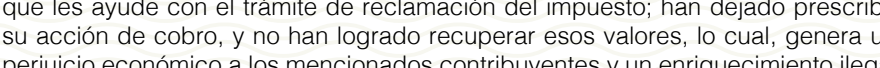
periucicio econonico
para el Estado

El princiiio de eficieincia por parte de las Administraciones Tributarias sev ve vulne-
rado cuando estas gastan recursos en atender tramites que pueden ser evitados - resueltos de una mejor forma; por tal razón, este principio se ha incluido
la legistación, con la finalidad de que cada entidad pública obtenga los meipes resultados en sus procesos, con la utilización de la menor cantidad de recursos manera, se resguardan los recursos económicos del stadado Para el presente cas de estudio, es evidente que los trámites de pago indebido de impuesto a la rent to éstos pueden ser evitados o resueltos con un processo más simplificado; cuyyo

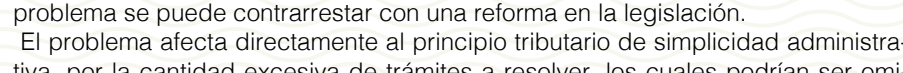

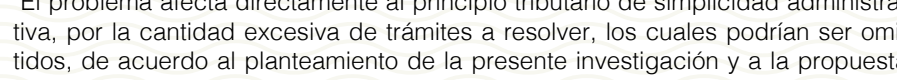

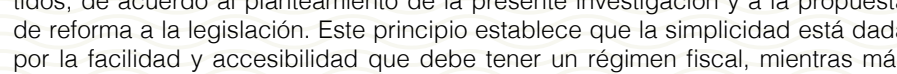
sencillo sea para el contribuyente presentar sus declaracioines, la actividad re
cauudadora cumplira su comerido. No obstante, una vez mas debemos aludir a echa de aceptación: 30 de Junio 2017 cultura y conciencia tributaria, no importa entonces cuán sencillo sea presents
una declaración, mientras esté arraigadal la intención de elusión o evasion (Casto Muñoz, 2010 ).

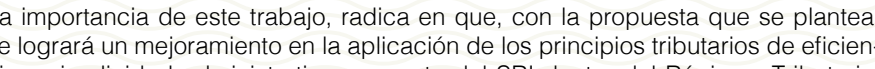
cia y simplicidad administratriva por parte del SRI dentro del Reginimen Tributiario

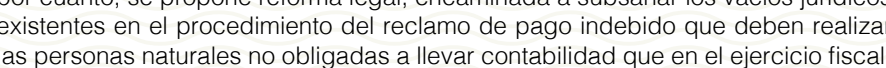

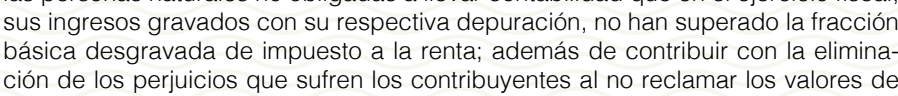
cion de los periucicios que surten los contribuyent
Inpuesto a la renta pagados en forma indebida.

En las conclusiones, como propuesta de la investigacionn, consta un procodimiento
simplificado a ser aplicado por el SRII para la devolución del impuesto a la renta Tha superado la fracción básica desgravada de la tabla correspondiente, as

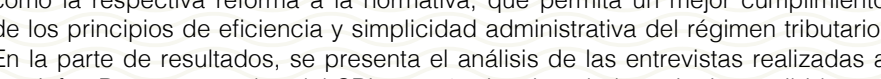
os Jefes Departamentales del SRI zona a, sintetizizando los criterios recilididos en Finalmente, en las conclusiones consta un analisis sobre el cumplimiento de los
principios de eficiencia y simplicidad adminisistrativa por parte del SRl. Existen varos trabajos en los que se realiza una conceptualizacion de los principios tributatacación de los princiiios de eficiencia y simplicidad administrtativa en los reclamos administrativivos, en lo que respecta a la devolución del impuesto a la renta por las
Retenciones en la Fuente, cuyys valores retenidos se constituyen en pago inde. bido. En cuanto a temas relacionados, se mencionanan los siguientes trabajos que
se han realizado: Simón Ortera (2011), Erazo (2012), Bautisita (2011), Masbernat

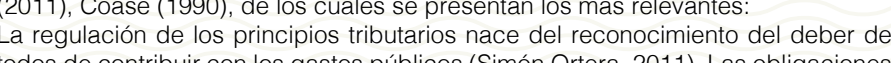
tributarias deben tener como caracterírítica fundamentalal el cumplimiento de los principios tributarios por parte del Estado, sin dejar de lado el deber de contribuil
con el gasto puiblico por parte de los contribuyentes; por lo que, las adminisistrser las pioneras en cumplir con los principioios, para que sus obligaciones sean ejecutadas de forma adecuada, respetando los derec
sin perjudicarlos en el ámbito legal, económico y social

El Ecuador cuenta con una estructura impositiva propia de un résimen tributario "sistema tributario", en razón de que todavia no existe una correlación entre sus

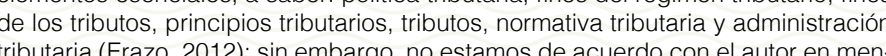
ción, ya que, nuestro pais si cuenta con una estructura impositiva propia de dun

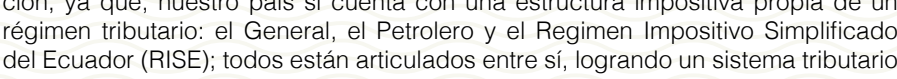
coherente y completeto; no obstante, éstos siempre serán perfectibles y cada vez
eccesitan de reformas que contribuyan a su perfeccionamiento, como las que se

Principios Juridicos Tributarios
Principio de Eficicincia

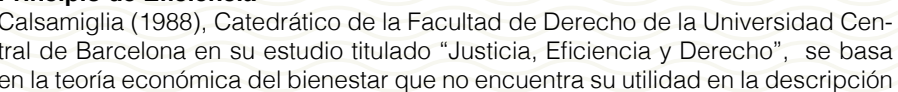
de la realidad sino en la luz que puede offecer para plantear problemas, para

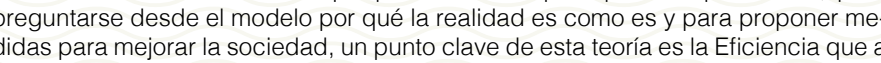
criterio de Wilfredo Pareto se pretendia resolver algunos de los inconvenientes del 